\section{Wie viele Kinder sind psychisch gestört?}

Psychische Störungen bei Kindern und Jugendlichen haben eine hohe Relevanz - ähnlich hoch wie Adipositas. Mithilfe der BELLA-Studie möchte das Robert-Koch-Institut mehr über das seelische Wohlbefinden und Verhalten von Kindern und Jugendlichen herausfinden.

$\mathrm{D}$ ie Ergebnisse zu den psychischen Auffälligkeiten wurden von Studienleiterin Professor Ulrike Ravens-Sieberer, Universitätsklinikum Hamburg-Eppendorf, vorgestellt: In Deutschland beträgt die Zahl der Kinder und Jugendlichen mit psychischen Auffälligkeiten rund $18 \%$. Nach den Ergebnissen der BELLA-Studie leiden 9,7\% sehr wahrscheinlich an einer psychischen Störung. Bei etwa 12,2\% der Kinder besteht möglicherweise eine psychische Auffälligkeit. Insgesamt liegen somit bei $21,9 \%$ zu- mindest Hinweise auf psychische Auffälligkeiten vor. „Ich möchte dies ausdrücklich relativieren: Wir haben eine stabile Rate von circa $10 \%$ bei Kindern und Jugendlichen, bei denen wir mit hoher Wahrscheinlichkeit von einer psychischen Störung sprechen können“, erklärte RavensSieberer. Daneben gibt es eine Gruppe von Kindern, bei der eine genauere Abklärung und Begleitung auch der Familie notwendig ist. Jungen sind mit $10,9 \%$ öfter betroffen als Mädchen (8,4\%). Die häufigsten psychi- schen Erkrankungen sind Ängste, die 10\% der Erkrankungen ausmachen und vor allem jüngere Kinder betreffen. Störungen des Sozialverhaltens sind mit 7,6\% vertreten. Depressionen kommen bei 5,4\% vor.

Eines der überraschendsten Ergebnisse der BELLA-Studie war für Ravens-Sieberer und ihre Kollegen, dass die Zahlen stark vom sozioökonomischen Status der Familien abhängig waren. Kinder aus Familien mit einem niedrigen sozioökonomischen Status waren häufiger von psychischen Störungen betroffen.

Dr. Nicola Zink

Ulrike Ravens-Sieberer, Verhaltensauffälligkeiten und psychische Probleme bei Kindern und Jugendlichen in Deutschland, im Rahmen der 108. Jahrestagung der Deutschen Gesellschaft für Kinder- und Jugendmedizin (DGKJ), Hamburg, 14.9.2012

\title{
Angiotensin-Rezeptorblocker könnten vor Alzheimer schützen
}

Bei Patienten, die mit Angiotensin-Rezeptorblockern behandelt werden, finden sich autoptisch seltener pathologische Befunde, die für die Alzheimerkrankheit typisch sind.

A us Tierversuchen ist bekannt, dass Angiotensin II die Amyloidproduktion ankurbelt. Dies legte für den Geriater Ihab Hajjar und sein Team die Vermutung nahe, dass eine Therapie mit Sartanen die Alzheimer-typischen Amyloidablagerungen verringern sollte.

In einer neuropathologischen Untersuchung sind die Forscher ihrer Hypo- these nachgegangen. Sie sammelten Befunde von 890 Autopsien hypertoner Patienten (Durchschnittsalter 81 Jahre), die zuvor Hinweise auf kognitive oder hirnpathologische Beeinträchtigungen gezeigt hatten. 133 von ihnen waren mit Sartanen, 577 mit anderen Antihypertensiva und 180 nicht medikamentös behandelt worden.

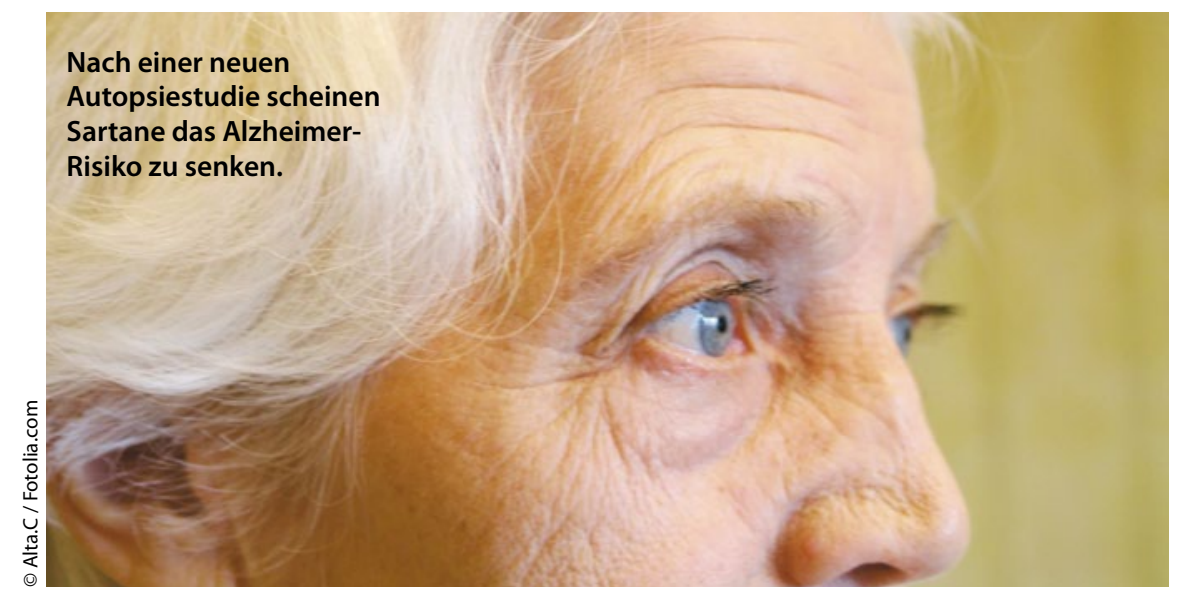

\section{$\mathbf{3 5} \%$ weniger Alzheimer-Diagnosen}

Die Wahrscheinlichkeit, dass die pathologische Untersuchung des Gehirns eine Alzheimer-Diagnose nach sich zog, war bei den mit Sartanen behandelten Patienten rund $35 \%$ niedriger als bei jenen, die ein Medikament aus einer anderen Antihypertensivagruppe erhalten hatten. Dasselbe Resultat zeigte der Vergleich mit den unbehandelten Hypertonikern. Die ermittelten Unterschiede erwiesen sich als statistisch relevant. Auch die Zählung neuritischer Plaques ergab für die Sartangruppe signifikant niedrigere Werte, selbst nachdem man etwa den ApoEStatus in die Kalkulation einbezogen hatte. Schlaganfälle fanden sich hingegen bei den Sartan-Behandelten häufiger als in den anderen Gruppen.

„Eine Therapie mit Angiotensin-Rezeptorblockern ist mit einer geringeren Rate Alzheimer-typischer Autopsiebefunde verbunden", resümieren Hajjar und Kollegen. Sie fordern weitere Untersuchungen, in denen dem Einfluss von Sartanen auf den kognitiven Verfall bei Demenz- beziehungsweise Alzheimer-Patienten nachgegangen werden soll.

Robert Bublak

Hajjar I et al. Impact of Angiotensin Receptor Blockers on Alzheimer Disease Neuropathology in a Large Brain Autopsy Series. Arch Neurol. Published online September 10, 2012; doi:10.1001/archneurol.2012.1010 